\title{
Parler en ville, Parler de la ville, Essais sur les registres urbains, Wald Paul et Leimdorfer François (dir.)
} Éditions UNESCO / Éditions de la Maison des sciences de l'homme, Paris 2004, 265 p., ISBN : 2-7351-0967-4

\section{Lamia Missaoui}

\section{(2) OpenEdition}

Journals

Édition électronique

URL : https://journals.openedition.org/remi/4278

DOI : $10.4000 /$ remi.4278

ISSN : $1777-5418$

Éditeur

Université de Poitiers

Édition imprimée

Date de publication : 1 juin 2005

Pagination : 167-170

ISBN : 2-911627-40-7

ISSN : 0765-0752

Référence électronique

Lamia Missaoui, «Parler en ville, Parler de la ville, Essais sur les registres urbains, Wald Paul et Leimdorfer François (dir.) », Revue européenne des migrations internationales [En ligne], vol. 21 - n² | 2005, mis en ligne le 01 octobre 2008, consulté le 15 avril 2022. URL : http://journals.openedition.org/remi/4278 ; DOI : https://doi.org/10.4000/remi.4278

Ce document a été généré automatiquement le 15 avril 2022.

(C) Université de Poitiers 


\section{Parler en ville, Parler de la ville, Essais sur les registres urbains, Wald Paul et Leimdorfer François (dir.)}

Éditions UNESCO / Éditions de la Maison des sciences de l'homme, Paris 2004, 265 p., ISBN : 2-7351-0967-4

\section{Lamia Missaoui}

\section{RÉFÉRENCE}

Parler en ville, Parler de la ville, Essais sur les registres urbains, Wald Paul et Leimdorfer François (dir.) Éditions UNESCO / Éditions de la Maison des sciences de l'homme, Paris 2004, 265 p., ISBN : 2-7351-0967-4

1 Cet ouvrage collectif est la troisième livraison de la collection les mots de la ville ${ }^{1}$ publiée par les Editions UNESCO et la Maison des Sciences de l'Homme.

2 Paul Wald et François Leimdorfer ont dirigé ce numéro qui offre une réflexion particulièrement enrichissante tant d'un point de vue scientifique que d'un point de vue heuristique. Cet ouvrage qui conjugue un ensemble de savoirs urbains de l'anthropologie et de la sociolinguistique, offre des textes d'une grande qualité tant pour les chercheurs, les étudiants, les administrateurs, que les autres professionnels de la ville. Cette rencontre entre l'anthropologie, la linguistique et la sociologie nous propose une promenade dans différents sites, différents horizons, différentes cultures nationales pour appréhender et dire la ville. La sociolinguistique est un élément central dans la compréhension des tensions entre habitats/habitants/aménageurs : c'est par le biais de l'emploi des mots de la ville que les contributions à cet ouvrage abordent la mise en discours de la ville et du caractère urbain des gens et des choses. "L'existence de cette catégorie des mots est un fait de discours plutôt qu'un fait de langue. En effet, son existence comme catégorie signifiante est tributaire non tant d'une sémantique lexicale stabilisée de la langue que de la régulation sociale du discours... » 
3 C'est pour cela que, dans cet ouvrage on considère tour à tour la ville d'une part comme un milieu exerçant ses effets propres sur les phénomènes sociaux, et d'autre part la ville comme objet d'actions, comme objets façonnés par des activités sociales. Pour étudier le fait urbain, et pour paraphraser les coordinateurs « ce doublet... » si on prend le soin de le lier permet de rompre ce qui est souvent étudié séparément. Ce doublet prenant l'anthropologie comme alliée de la sociolinguistique permet d'appréhender une question, un objet, ici la ville dans sa «totalité». Surtout parce qu'on y lie la complexité de la ville à partir de sa constante mouvance, son hétérogénéité, sa pluralité. "Parler en...» et "parler de..." permet justement d'accéder à ce qui d'ordinaire est étudié de façon séparée.

4 Quand nous précisons que Parler en ville, parler de la ville propose un savoir anthropologique urbain qui vise à appréhender une totalité c'est aussi parce que «parler de » et « parler en » sont deux caractéristiques centrées sur la dynamique des pratiques langagières liées aux phénomènes sociaux urbains. Ces deux caractéristiques permettent à partir de suite d'opérations individuelles, pratiques et empiriques, de faire remonter des informations théoriques des discours de la ville.

5 L'originalité de l'ouvrage se trouve dans le fait que les coordinateurs, conscients de cette difficulté d'appréhender la ville et surtout les discours de "la ville et en ville", ont posé des notions intermédiaires qui permettent de faire le passage de l'une à l'autre ("parler de " "parler en ») autrement dit le passage de l'empirique au théorique. Ces notions intermédiaires sont a priori distinctes, distantes, mais appréhendées simultanément, la majorité des textes de l'ouvrage les conjuguent afin de donner sens à la réalité des villes qu'ils traitent.

6 Ces notions phénoménologiques en somme concernent des moments d'émergences, d'affrontements et de parcours. Ces propositions intermédiaires sont, d'une part, posées comme équivalentes dans l'approche des faits sociaux et d'autre part, appréhendées en même temps dans leurs manifestations surtout pour décrire, penser empiriquement et théoriquement la ville dans une perspective à la fois sociolinguistique et anthropologique, centrée sur la dynamique des pratiques langagières.

7 Même si par commodité, l'ouvrage est divisé en trois parties, notre lecture n'y a vu que liens, continuité et complémentarité. L'unité de la triade est d'autant plus accentuée qu'un sociolinguiste de renommée, Pierre Achard, est très présent dans l'ouvrage et fait à son tour liens, consensus, surtout pour les coordinateurs, un fil conducteur théorique de la sociologie du langage.

8 La description de l'urbanité est présentée au travers d'études de cas très diverses tant dans les sites (différentes villes sont étudiées: Zagreb, Tunis, Buenos Aires, Naples); que différents pays: (la Zambie, l'Algérie, l'Inde...). Dépaysement, distanciation, comparaison, continuité sont alors les maîtres mots d'une réflexion qui évite l'ethnocentrisme ; le point de vue, d'ici et d'ailleurs, rend compte à la fois des pluralités du phénomène linguistique urbain et des continuités liées à des rythmes sociaux, des flux, des séquences, des mobilités : l'ordre des espaces et l'ordre des temporalités sont dans plusieurs textes indissociables. Ils permettent de relier les interactions dans des situations ou des contextes de l'activité quotidienne à des modes d'organisations fortement dépendantes d'un savoir être urbain. 
9 Chacun de ces sites propose une analyse des dynamiques des pratiques langagières liées aux phénomènes urbains. Ensuite c'est le caractère constitutif de ces discours qui font la ville: décrire la ville n'est pas une activité neutre, transparente, disjointe de la réalité à laquelle elle prétend référer, mais une activité structurante qui construit ces objets de discours par la façon dont elle les organise, les catégorise et les situe par rapport à d'autres.

10 Pour cela l'ouvrage reprend la réflexion sur le terme du « registre discursif» de l'urbain : «Registre» comme «lieu commun » permettant d'analyser des façons multiples dont différents acteurs disent la ville et contribuent ainsi à la façonner, à la faire changer, à lui donner sens et intelligibilité, à construire des pertinences et des évidences qui organisent les pratiques ordinaires et scientifiques... « ...En fait si l'on parle de registre, on entend aussi par ce terme un ensemble de textes "apparentés", associés à ces catégories de sources et leur activité (comme produire un texte savant, un ouvrage littéraire, un guide touristique, un rapport d'urbanisme, ou simplement parler en

ou parler de la ville)» Mais d'un autre côté "... si par "registre" on entend une modalité d'expression linguistique qui se développe avec d'autres, différentes, mais à l'intérieur du même système linguistique, nous devons comprendre dans quelle mesure, à Naples, les dénominations des espaces impliquent l'utilisation de termes différents dans le cadre de lexiques spécialisés (techniques, administratifs, sociaux,) ou bien s'il s'agit d'utilisations différentes de ces mêmes termes selon le locuteur et le contexte dans lequel le terme est utilisé» (texte de Alessandra Broccolini). Donc les discours sur la ville ou en ville sont des catégories d'usages perçues par les locuteurs, les acteurs de l'urbain comme des catégories communes voire évidentes.

11 L'»émergence » permet de rendre compte de la complexité de la ville: pour la sociolinguistique la ville est un véritable défi dans le sens où on est confronté à un lieu d'hétérogénéité qui permet de poser de façon emblématique la question de la variation, du changement, du contact de langue, à propos d'identités et de pratiques sociolangagières multiples et hybrides. Autrement dit, cette complexité fait référence aux dynamiques sociales et culturelles inscrites dans le temps et l'espace et qui agit sur les discours et les langues; le texte de Sylvie Teveny rend compte d'une façon remarquable de la production simultanée d'un espace urbain inédit dans la culture inuite et d'un lexique spécifique laissant peu de place aux emprunts. Ici le registre urbain fonctionne comme la part ostensible d'appropriation culturelle de valeurs importées.

12 Cette notion "d'émergence", au travers des différentes contributions exprime les transformations urbaines et ses effets sur les registres urbains. Les dialectiques de l'identité et de l'altérité contribuent indissociablement aux relations les plus manifestes lors des rencontres des diverses populations dans l'espace urbain. (texte de Dubravko Skiljan)

13 L'affrontement résume «un ensemble de décalage de pratiques langagières qui donnent lieu à la concurrence et au conflit des discours multiples « en ville et de la ville ». Cette notion permet d'appréhender les situations urbaines conflictuelles parce que l'appropriation ou la ré-approriation de l'espace via les pratiques discursives des groupes opposés devient un enjeu du conflit. Le texte de Moussaoui Abderrahmane montre comment des décisions d'aménagement urbain peinent à réussir voire échouent sans un aménagement linguistique raisonné. 

sociolinguistique de la ville est un véritable défi comme nous l'énoncions plus tôt: d'abord parce que au sein des études portant sur l'espace, dans la langue, la ville devient un objet de discours complexe entraînant de nombreux problèmes de formulation, d'articulation discursive, ensuite au sein même de la sociolinguistique, la ville est un lieu d'hétérogénéité qui permet de poser de façon emblématique la question de la variation, du changement, du contact de langue, de refus de langue, ce qui donne des identités et des pratiques socio-langagières multiples et hybrides.

\section{NOTES}

1. RIVIÈRE d'ARC (H), (éd.), 2001, Nommer les nouveaux territoires urbains et TOPALOV (C.), (éd.), 2002, Les divisions de la ville.

\section{AUTEURS}

\section{LAMIA MISSAOUI}

Laboratoire PRINTEMPS, Université de Versailles, Saint-Quentin-en-Yvelines 